\title{
Research on Stresses of Narrow Joints Caused by Construction Process of CRTSIISlab Track
}

\author{
Yongjian Zhu \\ MOE Key Laboratory of High-speed Railway Engineering, Southwest Jiaotong University, Chengdu 610031, China.
}

\begin{abstract}
A separate finite element model of 1/4 unit track slab of CRTS IIslab track was established for the analysis of the influence of construction process on stresses of narrow joints. When track slabs were connected by tension and when wide joints hardened, the impacts of the longitudinal connection of CRTS II slab on stresses of narrow joints were analysed. The results show that when track slabs are connected by tension, the bonding states between track slab and mortar layer have little effect on track slab expansion displacement and the re-compression of narrow joints caused by the tensioning force cannot be ensured. If track slabs are connected by tensioning force after the concrete hardening of narrow joints, compressive stresses of narrow joints can be increased by 3.03 MPa. Due to the influence of longitudinal connection of CRTSIIslab, the respective track slab temperature for wide joints hardening $T_{\mathrm{k}}$ and narrow joints hardening $T_{\mathrm{z}}$ are difficult to keep consistent. When wide joints hardened, it potentially makes the compressive stresses of narrow joints zero or very large. In order to keep the narrow joints compressed when wide joints hardened, the measures to control $T_{\mathrm{z}}$ and $T_{\mathrm{k}}$ should be taken to make $T_{\mathrm{k}}$ slightly higher than $T_{\mathrm{z}}$.
\end{abstract}

\section{Instruction}

CRTSIIslab track mainly consists of track slab, cement emulsified asphalt mortar layer, base plate or support layer. In addition to that the mortar layer, base plate or support layer is the longitudinal continuous structure, track slabs are also connected through joints between slabs to become a longitudinal continuous reinforced concrete structure. Since this structure was used in Beijing-Tianjin inter-city railway in 2008, the overall quality is in good condition, which meets the requirements of high-speed railway for high smoothness and high stability. However, the high-speed railway has been widely constructed in a variety of districts, in which the climatic conditions vary greatly. As a longitudinal continuous structure, the temperature effect of CRTSIIslab track caused by temperature load is very significant. Affected by the environment, temperature, temperature gradient and so on, the gap under slabs was generated, and joint-defects and arch between slab joints appeared. It brought not only maintenance and repair work, but also adverse effects to normal operation. The uneven stresses in joints are an important cause leading to track slab arch and joint-defects. Therefore, it is very necessary to study the mechanical characteristics of joints between slabs.

In addition to the influence of construction quality, joint-defects and joint-deformation between slabs were mainly influenced by temperature. At present, there have been many researches on behaviour of joint-defects, the stress and deformation and dynamic response of track structure caused by joint-defects [1-3]. However, there were few studies on influences of construction process ofIIslab on joint stresses. Through the analysis of mechanical relationships between track slab and mortar layer, steel bars, joints, the finite element model was established. The influences of construction process ofIIslab under the action of temperature force on stresses of narrow joints were analysed.

\section{Longitudinal connection of CRTS II slab}

CRTS II track slabs are prefabricated structure in the factory. After the laying of track slabs, they are connected to become a longitudinal continuous structure through joints between slabs. Joints between slabs are shown in Figs. 1 2. Six steel bars with a diameter of 20 $\mathrm{mm}$ connect two adjacent track slabs together by the tensioning slack adjusters at the end of slabs. According to the construction process [4], joints between slabs are poured firstly after the strength of emulsified asphalt cement mortar (CA mortar) under slab reaches $7 \mathrm{MPa}$, which is known as narrow joints with a height of $0.1 \mathrm{~m}$; When the strength of CA mortar reaches $9 \mathrm{MPa}$ and the concrete strength of narrow joints reaches $20 \mathrm{MPa}$, the six longitudinal steel bars will be connected by the tensioning slack adjusters, and each bar has a tensile load of $50 \mathrm{kN}$. Then the rest of joints will be poured at last, which are known as wide joints. The narrow joints

*Corresponding author: ${ }^{\text {a }}$ Yongjian Zhu,zyjian1987@ 163.com 
consist of three parts, whose lengths are about $0.2 \mathrm{~m}$, $0.4 \mathrm{~m}$, and $0.2 \mathrm{~m}$ respectively [4].

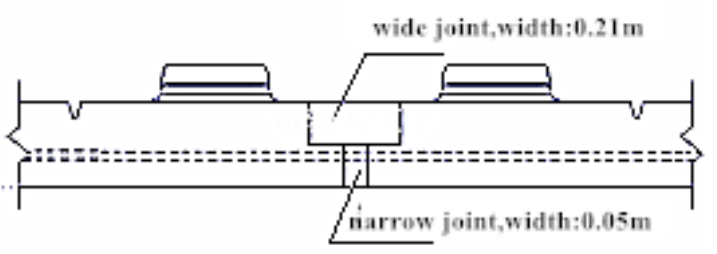

Figure 1. Picture of joints between slabs.

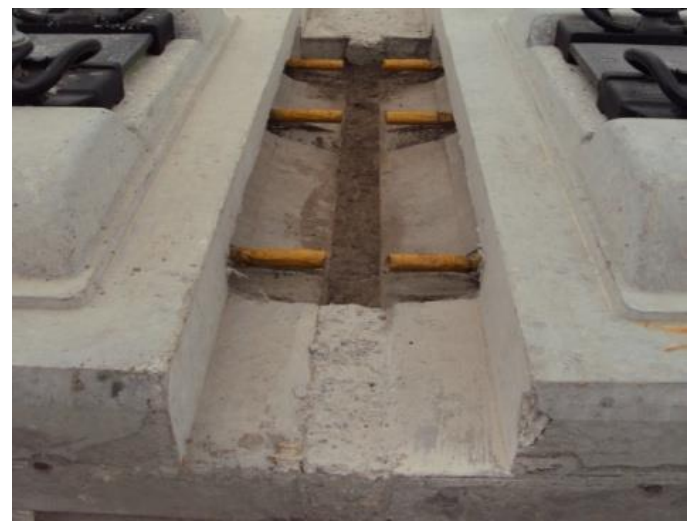

Figure 2. Narrow joints which had been Finished.

Judging from the construction process, compared with the continuous pouring of reinforced concrete structure, the concrete of narrow joints of CRTS II slab are poured firstly, then the concrete of wide joints. Therefore, there is a difference in slab temperatures when the narrow joints and wide joints hardened, and there is also a pre-tension when the longitudinal steel bars are connected. So the longitudinal connection characteristic of CRTSIIslab track is very obvious. When wide joints hardened, this characteristic made the stresses of narrow joints very complicated, which could be zero or very large. Zhou Min et al.'s research [5] shows that there is indeed some phenomenon on site that when the crack between wide joints and slabs is inexistent, there are cracks between narrow joints and slabs at the same place. This is in conflict with the design concept of II slab, which is "the compressive stresses of narrow joints should be always existent when track slabs are connected completely or the wide joints harden". It shows that some improvements are needed for current construction process control measures, so it is of certain engineering significance to carry out the research.

\section{Models and parameters}

\subsection{Model assumption}

(1) The deformation and stress of track slab are of symmetric distribution under the loads of longitudinal tension, joints between slabs and temperature.
(2) The displacements of track slab cross-section are evenly distributed.

(3) The stresses and strains of steel bars cross-section are evenly distributed.

(4) The stresses of narrow joints cross-section are evenly distributed.

(5) Considering that the interfaces between the concrete of joints and the end face of track slab are newold concrete joint surfaces, and the end face of track slab is vertical and its roughness is small, the interface bond stresses are small. Therefore the tensile restraints from the concrete of joints on track slab are neglected.

\subsection{Finite element model}

Track slab is simulated by solid element solid45.Its length, breadth, and height are $6.45 \mathrm{~m}, 2.55 \mathrm{~m}$, and $0.2 \mathrm{~m}$ respectively. Its elastic modulus is $3.60 \times 10^{4} \mathrm{MPa}$. Its Poisson's ratio is 0.2 . Its thermal expansion coefficient is $1.0 \times 10^{-5}$. The top width, bottom width, and height of pre-splitting crack are $38 \mathrm{~mm}, 4 \mathrm{~mm}$ and $28 \mathrm{~mm}$ respectively. The constraints of mortar layer and steel bars on track slab are simulated by ANSYS 3D nonlinear spring element COMBIN39, which parameters are shown in sections 2.3 and 2.4 respectively. The longitudinal and horizontal steel bars are simulated by LINK1 and LINK8 respectively. Its elastic modulus is $2.0 \times 10^{5} \mathrm{MPa}$. Its thermal expansion coefficient is $1.2 \times 10^{-}$ 5 . The length of horizontal common steel bars is $2.45 \mathrm{~m}$, and that of horizontal pre-stressed steel bars is $2.55 \mathrm{~m}$. The lengths of longitudinal steel bars are $6.2 \mathrm{~m}$ and $6.35 \mathrm{~m}$ in the positions of wide and narrow joints respectively. The length of tensioned rebar is $6.45 \mathrm{~m}$. The number and locations of steel bars are seen in literature [7].

Narrow joint is simulated by the bar element LINK10 with only compression. The differences in the cross section of joints are neglected. According to the symmetry of the model, a finite element model of $1 / 4$ track slab was established.

In order to consider the constraints of mortar on track slab at joints, the bottom thickness of track slab at wide joints is kept as $20 \mathrm{~mm}$. The final separate finite element model of 1/4 track slab is shown in Fig. 3.

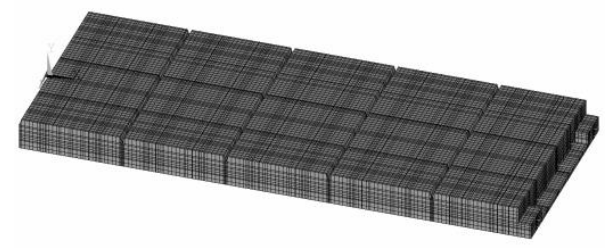

Figure 3. Finite element model of $1 / 4$ track slab.

\subsection{Bond slip behavior between track slab and tensioned rebar}

The steel bars, which are adopted by CRTSII track slab, all are hot rolled ribbed steel bars [7]. Code for design of concrete structures (GB50010-2010) provides the constitutive relation of the bond stress-slip between concrete and hot rolled ribbed steel bar. For CRTS II 
track slab, whose axial tensile stress $f_{\mathrm{t}, \mathrm{r}}$ is $3.55 \mathrm{MPa}$ [8], the value is not quite different from the average value of tensile strength of C55 concrete, which is $3.60 \mathrm{MPa}$ [9]. Then, there are $\tau_{\mathrm{cr}}=2.5 f_{\mathrm{t}, \mathrm{r}}, \tau_{\mathrm{u}}=3 f_{\mathrm{t}, \mathrm{r}}, \tau_{\mathrm{r}}=f_{\mathrm{t}, \mathrm{r}}, s_{\mathrm{cr}}=0.025 \mathrm{~d}$, $s_{\mathrm{u}}=0.04 \mathrm{~d}, s_{\mathrm{r}}=0.55 \mathrm{~d}$ [9], where $\mathrm{d}$ is the diameter of steel bar. Taking the tensioned rebar as an example, the relationship between bond stress $\tau_{\mathrm{s}}$ and relative slip displacement $s$ between track slab and tensioned rebar is shown in Fig. 4.

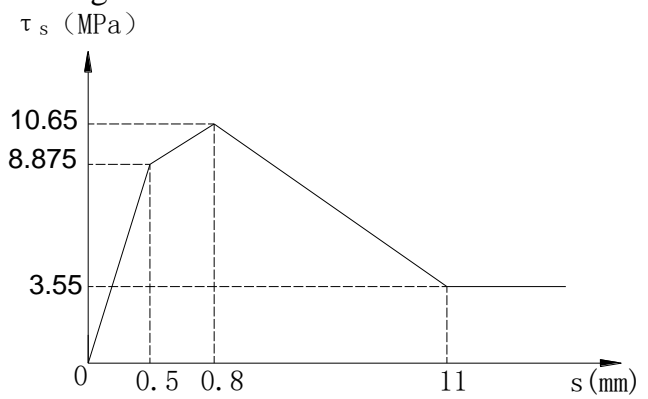

Figure 4. Bond slip curve between track slab and tensioned rebar.

For the rest of steel bars with a diameter of $8 \mathrm{~mm}$, $10 \mathrm{~mm}$ and $16 \mathrm{~mm}$ respectively, the corresponding slip curves can be obtained by calculating the corresponding $s_{\mathrm{cr}}, s_{\mathrm{u}}$, and $s_{\mathrm{r}}$.

\subsection{Friction characteristics between track slab and mortar layer}

The constraint of mortar layer on track slab was influenced by ambient temperature, and gradually decreased with time. Through analysis of the indoor and field tests between track slab and mortar layer at home, the bonding states between track slab and mortar layer are divided into three stages, which are good bond, partial bond and poor bond respectively, and will be simulated by a piecewise linear model.

When mortar layer was stiffened, the bonding state between track slab and mortar layer was little influenced by the environment. At this time, the interlayer state was regarded as good bond, and the selection of parameters were based on the indoor tests. When the bonding state between track slab and mortar layer is in good bond condition, the relative displacement between track slab and mortar layer was very small before the micro cracks were generated. It is considered that the angle of shear is directly proportional to the shear stress. When the bonding state between track slab and mortar layer is good bond, the inter-laminar shear stiffness $k_{\mathrm{c}}$ is:

$$
\begin{gathered}
k_{\mathrm{c}}=G_{\mathrm{c}} / h_{\mathrm{c}} \\
G_{\mathrm{c}}=E_{\mathrm{c}} / 2 /\left(1+v_{\mathrm{c}}\right)
\end{gathered}
$$

Where $E_{\mathrm{c}^{-}}$elastic modulus of mortar, $8000 \mathrm{MPa}$; $v_{\mathrm{c}^{-}}$Poisson's ratio of mortar material,0.2;

$h_{\mathrm{c}^{-}}$thickness of mortar layer, $0.03 \mathrm{~m}$ 。

It is known from the indoor shear tests between track slab and mortar layer $[2,11]$ that, the inter-laminar shear strength of $28 \mathrm{~d}$ age is about $0.44 \sim 0.78 \mathrm{MPa}$. When the model simulation was analyzed, the parameter was taken as $0.5 \mathrm{MPa}$ in most of the model. When the bonding state between track slab and mortar layer is in good bond condition, the inter-laminar shear strength is $0.5 \mathrm{MPa}$.

In the plate pushing test in China, the temperature of mortar was controlled at $20 \pm 2^{\circ} \mathrm{C}$ after mortar perfusion. The push plate force was about 120t, while the corresponding longitudinal shear stress was about $72 \mathrm{KPa}$, and the corresponding inter-laminar displacement was about $0.02 \mathrm{~mm}$ [10]. At this time, the bonding state between track slab and mortar layer was less affected by external environment, and the interlayer state was regarded as partial bond.

In the plate pushing test in German, the plate pushing force was about 41t, while the corresponding longitudinal shear stress was about $24 \mathrm{KPa}$, and the corresponding inter-laminar displacement was about $0.5 \mathrm{~mm}$ [12]. At this time, the bonding state between track slab and mortar layer was greatly influenced by external environment, and the interlayer state was regarded as poor bond.

In summary, the relationship between shear strength $\tau_{\mathrm{c}}$ and relative displacement $y_{\mathrm{bc}}$ between track slab and mortar layer was obtained as shown in Fig. 5.

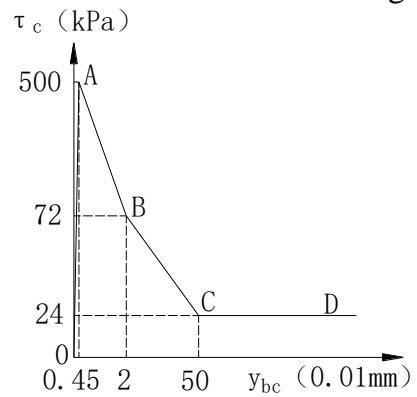

Figure 5. Relation ship of friction between track slab and mortar layer.

In Fig. 5, A, B and C points are the critical points of good bond, partial bond and poor bond respectively. The curve $0 A B C D$ is a weakening curve of the bonding state between track slab and mortar layer when environmental factors are considered.

\subsection{Boundary conditions}

(1) Displacement constraints were adopted at the end of narrow joints, and the values of displacement constraints took the displacement values of common nodes between track slab and narrow joints when narrow joints hardened.

(2) The shear constraints of mortar layer on track slab were simulated by springs, and the bottom was fully constrained.

(3) Except degree of freedom along the length of steel bars, the remaining two degrees of freedom were restrained, only allowing the steel bars to expand freely along the length direction.

(4) Displacement constraints were adopted at the end of tensioned rebar after track slabs were connected by tension. The values of displacement constraints took the end displacements of tensioned rebar calculated by the corresponding working condition. 


\section{Result analysis}

Narrow joints are mainly affected by temperature expansion of track slab. The overall temperature rise and fall of track slab were mainly considered. From the mortar layer hardening to the concrete hardening of wide joints, the variation of track slab temperature was mainly affected by monthly temperature difference. For calculation the variation of track slab temperature is $30^{\circ} \mathrm{C}$, and the maximum of one-way temperature is $20^{\circ} \mathrm{C}$.

\subsection{Track slabs connected by tension}

The main influence factors of stresses of narrow joints are: the maximum of track slab temperature rise or fall $\Delta T_{\max }$ before concrete hardening of narrow joints, the ascending range of track slab temperature $\Delta T_{\mathrm{sl}}$ from concrete hardening of narrow joints to the connection between track slabs, and the tensioning force.

The initial interlayer states between track slab and mortar layer were divided into three working conditions: good bond, partial bond and poor bond. The corresponding interlayer weakening curves are OABCD, OBCD and OCD respectively in Fig 5.

When the stresses of narrow joints were analysed, the initial interlayer state between track slab and mortar layer was regarded as in good bond condition, and the corresponding interlayer weakening curve is OABCD in Fig 5. When calculating stresses of narrow joints, assuming that the narrow joints hardened after $\Delta T_{\max }$ was exerted on the model, and then $\Delta T_{\mathrm{sl}}$ and the tensioning force were exerted on the model. When analysing the effect of tensioning force on narrow joints, the above results were applied to subtract results of the model which exerted $\Delta T_{\max }$ and $\Delta T_{\mathrm{sl}}$ simultaneously. In the calculation, $\Delta T_{\max }$ are $0^{\circ} \mathrm{C}, 4^{\circ} \mathrm{C}, 5^{\circ} \mathrm{C}, 6^{\circ} \mathrm{C}, 7^{\circ} \mathrm{C}, 10^{\circ} \mathrm{C}, 20^{\circ} \mathrm{C}$ respectively, and $\Delta T_{\mathrm{sl}}$ are $5^{\circ} \mathrm{C}, 10^{\circ} \mathrm{C}, 20^{\circ} \mathrm{Crespectively.}$ Relationships between end displacements of track slab $y_{\mathrm{bc}}$ and $\Delta T_{\max }$ are shown in Fig. 6 . The increments of end displacements of track slab caused by the tensioning force $\Delta y_{\text {bd }}$ are shown in Fig. 7. Compressive stresses of narrow joints caused by tensioning force $\sigma_{\mathrm{zl}}$ are shown in Fig. 8. Relationships between compressive stresses of narrow joints $\sigma_{\mathrm{z}}$ caused by $\Delta T_{\mathrm{sl}}$ and $\Delta T_{\max }$ are shown in Fig. 9.

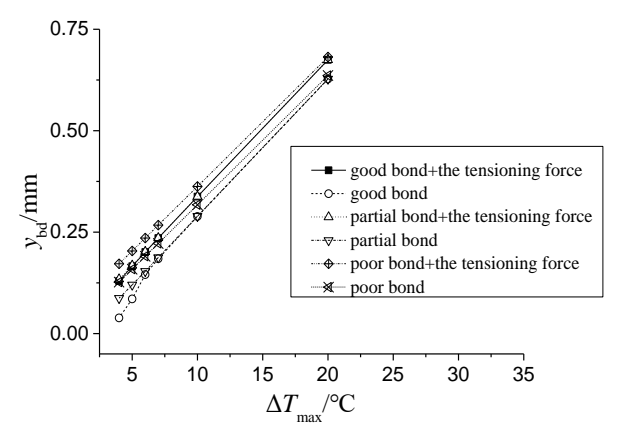

Figure 6. Relationships between end displacements of track slab ybd and $\Delta T_{\max }$.

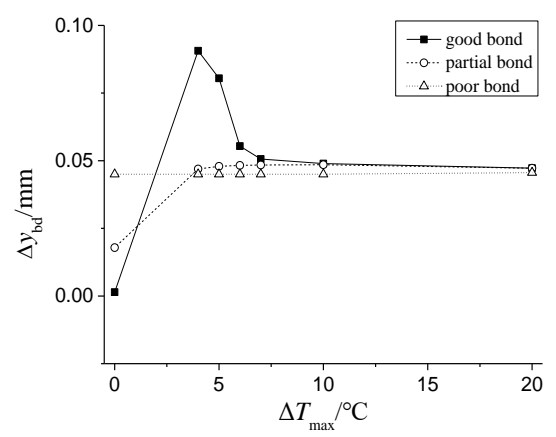

Figure 7. The increments of end displacements of track slab $\Delta y_{\text {bd }}$ caused by tensioning force.

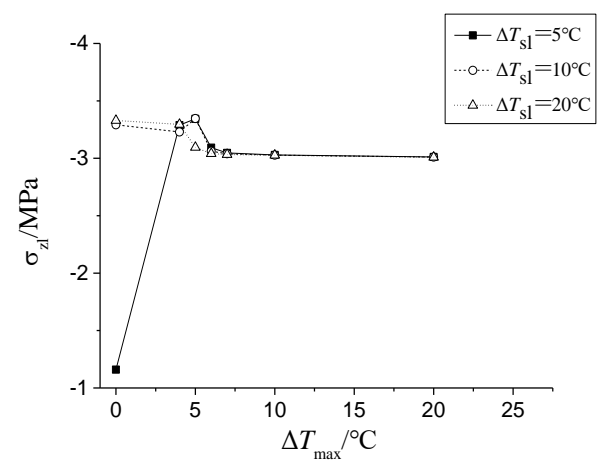

Figure 8. Compressive stresses of narrow joints $\sigma_{\mathrm{zl}}$ caused by tensioning force.

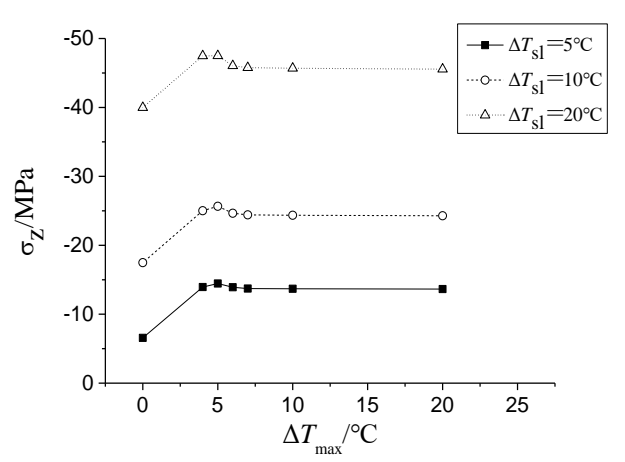

Figure 9. Relationships between compressive stresses of narrow joints $\sigma_{\mathrm{z}}$ caused by $\Delta T_{\mathrm{sl}}$ and $\Delta \mathrm{Tmax}$.

Figure 6 shows that with the increase of $\Delta T_{\max }$, end displacements of track slab $y_{\text {bd }}$ increase gradually. Combined with Fig. 5, it is not difficult to see that the bonding states between track slab and mortar layer are weakening gradually. As the initial interlayer states between track slab and mortar layer changing from good bond to partial bond or poor bond, $y_{\text {bd }}$ increase slightly, and the increments of $y_{\text {bd }}$ decrease gradually with the increase of $\Delta T_{\max }$. When $\Delta T_{\max }$ increases from $4^{\circ} \mathrm{C}$ to $20^{\circ} \mathrm{C}$, the increment of $y_{\text {bd }}$ decreases from $0.048 \mathrm{~mm}$ or $0.088 \mathrm{~mm}$ to $0.000088 \mathrm{~mm}$ or $0.0086 \mathrm{~mm}$ as the initial interlayer state between track slab and mortar layer changing from good bond to partial bond or poor bond. When $\Delta T_{\max }>6^{\circ} \mathrm{C}$, the maximum increment of $y_{\mathrm{bd}}$ is 
about $0.0029 \mathrm{~mm}$ or $0.038 \mathrm{~mm}$ as the initial interlayer state between track slab and mortar layer changing from good bond to partial bond or poor bond. When considering tensioning force, the increments of $y_{\mathrm{bd}}$ decrease slightly. It is indicated that $y_{\mathrm{bd}}$ are mainly related to the temperature rise and fall of track slab, and the bonding states between track slab and mortar layer has little effect on track slab expansion displacement.

Figure 7 shows that under the action of tensioning force, the end displacements of track slab increase, and the increments $\Delta y_{\text {bd }}$ are related to the interlayer bonding states. When the interlayer state is the best, namely $\Delta T_{\max }=0^{\circ} \mathrm{C}, \Delta y_{\text {bd }}$ increase gradually as the initial interlayer state between track slab and mortar layer changing from good bond to poor bond. When the interlayer state is weakened, namely $\Delta T_{\max }=4 \sim 20^{\circ} \mathrm{C}$, the tensioning force will accelerate interlayer weakening, which leads to the release of energy accumulated in track slab. And the better the interlayer bonding state, the more the release of energy. At the same time, $\Delta y_{\mathrm{bd}}$ decreases gradually as the initial interlayer state between track slab and mortar layer changing from good bond to poor bond, and then tend to be constant. When $\Delta T_{\max }>6^{\circ} \mathrm{C}, \Delta y_{\text {bd }}$ caused by the tensioning force is $0.047 \sim 0.051 \mathrm{~mm} 、 0.047 \sim 0.048 \mathrm{~mm}$ and $0.045 \sim 0.046 \mathrm{~mm}$ respectively as the initial interlayer state changing from good bond to poor bond.

Figure 8 shows that under the action of tensioning force, compressive stresses of narrow joints increase. When the interlayer state is the best, namely $\Delta T_{\max }=$ $0^{\circ} \mathrm{Cand} \Delta T_{\mathrm{sl}}=5^{\circ} \mathrm{C}$, tensioning force has the constraint of mortar layer on track slab strengthened, which leads to the small increment of compressive stresses of narrow joints $\sigma_{\mathrm{zl}}$. When the interlayer state is weakened, tensioning force will lead to the release of energy accumulated in track slab, which leads to $\sigma_{\mathrm{zl}}$ very large; When $\Delta T_{\max }=4 \sim 5^{\circ} \mathrm{C}$, the release of energy accumulated in track slab reaches the maximum, the maximal value of $\sigma_{\mathrm{zl}}$ is about 3.3 MPa. Then with the weakening of interlayer states, the release of energy accumulated in track slab decreases gradually, and then $\sigma_{\mathrm{zl}}$ tend to be constant. When $\Delta T_{\max }>6^{\circ} \mathrm{C}, \sigma_{\mathrm{zl}}$ caused by tensioning force is about 3.01 3.05MPa, and the change rule of $\sigma_{\mathrm{zl}}$ is basically the same as the change rule of solid line in Fig. 7.

Figure 9 shows that with the increase of $\Delta T_{\mathrm{sl}}$, compressive stresses of narrow joints increase gradually. When the interlayer state is the best, namely $\Delta T_{\max }=0^{\circ} \mathrm{C}$, the constraint of mortar layer on track slab is strengthened gradually with the increase of $\Delta T_{\mathrm{sl}}$, and $\sigma_{\mathrm{z}}$ gets to the minimum values. When the interlayer state is weakened, $\Delta T_{\mathrm{sl}}$ leads to the release of energy accumulated in track slab. When $\Delta T_{\max }=4 \sim 5^{\circ} \mathrm{C}$, the release of energy accumulated in track slab reaches the maximum, and $\sigma_{\mathrm{z}}$ reaches the maximum. Then with the weakening of interlayer states, the release of energy accumulated in track slab decreases gradually, and $\sigma_{z}$ decreases gradually and then tends to be constant. When $\Delta T_{\max }>6^{\circ} \mathrm{C}, \sigma_{\mathrm{z}}$ is mainly related to $\Delta T_{\mathrm{sl}}$. When $\Delta T_{\mathrm{sl}}$ are $5^{\circ} \mathrm{C} 、 10^{\circ} \mathrm{Cand} 20^{\circ} \mathrm{Crespectively,} \sigma_{\mathrm{z}}$ are $13.65 \sim 13.73$ $\mathrm{MPa}$ 、 $24.28 \sim 24.41 \mathrm{MPa}$ and $45.55 \sim 45.77 \mathrm{MPa}$ respectively. The change rule of $\sigma_{\mathrm{z}}$ is basically the same as the change rule of solid line in Fig. 7.

$\Delta T_{\max }$ is the maximum of track slab temperature rise or fall before the concrete hardening of narrow joints, which is mainly related to diurnal temperature difference and should be more than half the maximum of that. The diurnal temperature difference is usually $10 \sim 20^{\circ} \mathrm{C}$, so the possibility that $\Delta T_{\max }$ is less than $6^{\circ} \mathrm{C}$ is very small. Therefore, the interlayer bonding state had been very weak when track slabs were connected by tensioning force. When $\Delta T_{\max }>6^{\circ} \mathrm{C}$, is about $0.045 \sim 0.051 \mathrm{~mm}$, and $\sigma_{\mathrm{zl}}$ is about $3.01 \sim 3.05 \mathrm{MPa}$. Also $\sigma_{\mathrm{z}}$ is mainly related to $\Delta T_{\text {sl. }}$. When $\Delta T_{\text {sl }}$ is $5^{\circ} \mathrm{C} \sim 20^{\circ} \mathrm{C}, \sigma_{\mathrm{z}}$ is about $13.65 \sim 45.77$ $\mathrm{MPa}$.

End displacements of track slab caused by the tensioning force are very small. If track slab is cooled down and contracted after the concrete hardening of narrow joints, the pre-compression of narrow joints caused by tensioning force cannot be ensured. Therefore, when track slabs are connected by tensioning force, narrow joints may not be stressed, or they may be subjected to large compressive stresses due to larger $\Delta T_{\mathrm{sl}}$.

\subsection{Wide joints hardened}

From the connection between track slabs to concrete hardening of wide joints, the temperature expansion of track slab is affected by mortar layer, narrow joints and tensioned rebar. The main influence factors of stresses of narrow joints are $\Delta T_{\max }$, track slab temperature $T_{1}$ when track slabs are connected by tension, track slab temperature $T_{\mathrm{z}}$ when narrow joints hardened, track slab temperature $T_{\mathrm{k}}$ when wide joints harden.

For calculation simplification, it is assumed that the narrow joints harden when track slabs are connected by tension and $T_{1}=\Delta T_{\max } . \Delta T_{\max }$ are $0^{\circ} \mathrm{C},-4^{\circ} \mathrm{C},-5^{\circ} \mathrm{C},-6^{\circ} \mathrm{C}$,$7^{\circ} \mathrm{C},-10^{\circ} \mathrm{Crespectively,} \mathrm{the} \mathrm{corresponding} \mathrm{values} \mathrm{of}$ displacement constraints at the end of tensioned rebar are $0.250235 \mathrm{~mm}, 0.377998 \mathrm{~mm}, 0.416053 \mathrm{~mm}, 0.451592 \mathrm{~mm}, 0$ $.486573 \mathrm{~mm}, 0.590754 \mathrm{~mm}$ respectively. $T_{\mathrm{k}}$ are $5^{\circ} \mathrm{C}, 10^{\circ} \mathrm{C}, 15^{\circ} \mathrm{C}, 20^{\circ} \mathrm{Crespectively.} \mathrm{After} \Delta T_{\max }$ and the tensioning force were exerted to the model, narrow joints began to bear force, and then $T_{\mathrm{k}}$ was exerted on the whole model. When wide joints harden, the end displacement of track slab $y_{\mathrm{bc}}$ is shown in Fig.10, and compressive stresses of narrow joints $\sigma_{\mathrm{z}}$ are shown as Fig. 11.

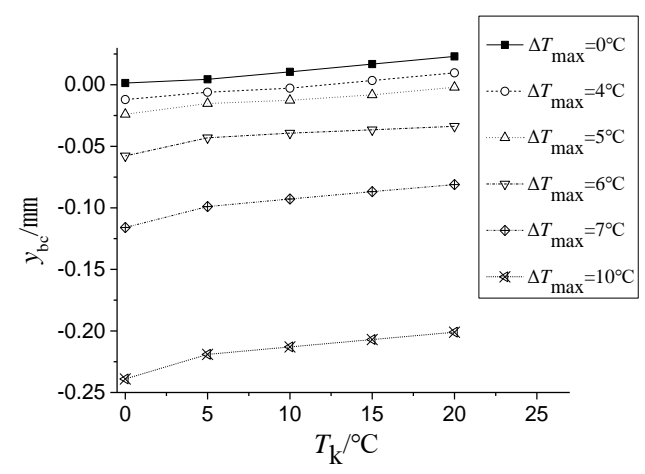

Figure 10. End displacements of track slab $y_{\mathrm{bc}}$ when wide joints harden. 


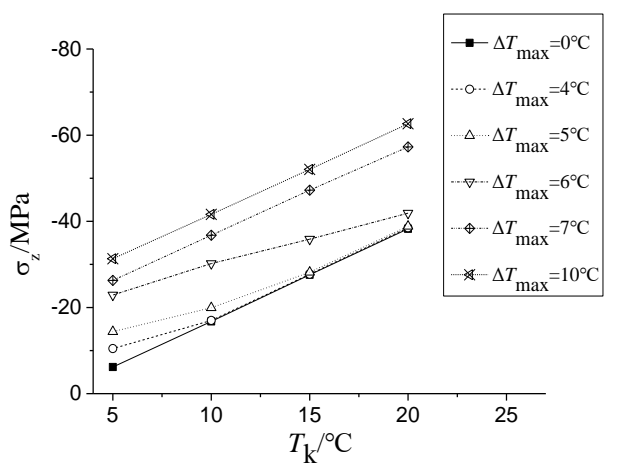

Figure 11. Compressive stresses of narrow jionts $\sigma_{\mathrm{z}}$ when wide joints harden.

Figure 10 shows that with the increase of $T_{\mathrm{k}}$, end displacements of track slab $y_{\mathrm{bc}}$ increase gradually, but the increments are small. When $T_{1}=\Delta T_{\max }$ is $0 \sim-10^{\circ} \mathrm{Cand}$ $T_{\mathrm{k}}$ is $20^{\circ} \mathrm{C}$, namely the temperature rise amplitude of track slab $\Delta T_{\mathrm{z}}$ is $20 \sim 30^{\circ} \mathrm{Cafter}$ the concrete hardening of narrow joints, the increment of $y_{\mathrm{bc}}$ is about $0.02157 \sim 0.03851 \mathrm{~mm}$.

Figure 11 shows that with the increase of $T_{\mathrm{k}}, \sigma_{\mathrm{z}}$ increase gradually. When $\Delta T_{\max }>6^{\circ} \mathrm{C}, \sigma_{\mathrm{z}}$ increase linearly. Because the possibility that $\Delta T_{\max }$ is less than $6^{\circ} \mathrm{C}$ is very small. When $\Delta T_{\max }>6^{\circ} \mathrm{C}$, the relationship between $\sigma_{\mathrm{z}}(\mathrm{MPa})$ and $\Delta T_{\mathrm{z}}\left({ }^{\circ} \mathrm{C}\right)$ is fitted to linear formula (3), and in this formula: $\Delta T_{\mathrm{z}}=T_{\mathrm{k}}-T_{1}$.

$$
\sigma_{\mathrm{z}}=-2.13 \Delta T_{\mathrm{z}}
$$

When considering the tensioning force, -3.03 should be added to the formula (3). Therefore, $\Delta T_{\mathrm{z}}$ in the formula (3) can be approximately $\left(T_{\mathrm{k}}-T_{\mathrm{z}}+1.42\right)$.

Extension displacements at the end of track slab will become very small after the concrete hardening of narrow joints. The compressive stresses of narrow joints caused by tensioning force are only equivalent to the effect of temperature rise $1.42^{\circ} \mathrm{Cof}$ track slab after the concrete hardening of narrow joints. If $\left(T_{\mathrm{z}}-T_{\mathrm{k}}\right)$ is more than $2^{\circ} \mathrm{C}$, narrow joints may not be stressed when wide joints hardened, and the narrow joints may not be stressed during the operation forever. This analysis result is also supported by the survey of literature [5], and which is contrary to the design concept of CRTSIIslab.

If the constraints of steel bars, pre-splitting cracks and mortar layer on track slab are ignored, the relationship between $\sigma_{\mathrm{z}}$ and temperature rise amplitude of track slab $\Delta T$ after the concrete hardening of narrow joints was deduced in formula (4).

$$
\sigma_{\mathrm{z}}=-2.20 \Delta T
$$

Compared with formula (3), $\sigma_{\mathrm{z}}$ increases by $3.2 \%$. Therefore, $\sigma_{\mathrm{z}}$ is mainly related to $\Delta T$.

If $T_{\mathrm{k}}>T_{\mathrm{z}}$, when $\Delta T_{\mathrm{z}} \geq 12^{\circ} \mathrm{C}, \sigma_{\mathrm{z}}$ will exceed the compressive strength design value $25.3 \mathrm{MPa}$ of concrete, and narrow joints have the possibility of crushing.

In construction of joints between track slabs, it is required that the environment temperature is not more than $25^{\circ} \mathrm{C}$ during the construction of narrow joints, and wide joints should be constructed at the lowest temperature of the day and the environment temperature should be not more than $25^{\circ} \mathrm{C}[4]$. In fact, $\sigma_{\mathrm{z}}$ is mainly determined by the difference of track slab temperatures when wide joints and narrow joints hardened (namely $T_{\mathrm{k}}-T_{\mathrm{z}}$ ) and the tensioning force. In order to keep compressive stresses of narrow joints existent when wide joints hardened, it is not enough to control the pouring temperatures of joints only, we should also take measures to control $T_{\mathrm{k}}$ and $T_{\mathrm{z}}$. Furthermore, when track slab temperature is high, in order to $\operatorname{keep} \sigma_{\mathrm{z}}$ not too large, we should also make $T_{\mathrm{k}}$ slightly higher than $T_{\mathrm{z}}$.

\section{Conclusions}

(1) When track slabs are connected by tension, the interlayer bonding states between track slab and mortar layer have little effect on track slab expansion displacement. If the tensioning force is applied on model after the concrete hardening of narrow joints, the increments of end displacements of track slab is about $0.045 \sim 0.051 \mathrm{~mm}$. The increments of compressive stresses of narrow joints is about $3.01 \sim 3.05 \mathrm{MPa}$.

(2) When wide joints hardened, if track slab temperature for wide joints hardening $T_{\mathrm{k}}$ is less than track slab temperature for narrow joints hardening $T_{\mathrm{z}}$ over $2^{\circ} \mathrm{C}$, narrow joints may not be stressed and may not be stressed during the operation forever, which is contrary to the design concept of CRTSIIslab. With the increase of $T_{\mathrm{k}}$, compressive stresses of narrow joints $\sigma_{\mathrm{z}}$ increase gradually, and the relationship between $\sigma_{\mathrm{z}}$ and $\Delta T_{\mathrm{z}}$ is $\sigma_{\mathrm{z}}=-2.13 \Delta T_{\mathrm{z}}$ where $\Delta T_{\mathrm{z}}=T_{\mathrm{k}}-T_{\mathrm{z}}+1.42$.

(3) We should take measures to control $T_{\mathrm{k}}$ and $T_{\mathrm{z}}$, and make $T_{\mathrm{k}}$ slightly higher than $T_{\mathrm{z}}$.

\section{References}

1. P Wang, $\mathrm{H} \mathrm{Xu}, \mathrm{R}$ Chen et al Effects Analysis of Cracking of CRTSII Slab Track on Subgrade. J Southwest Jiaotong Univ 47,6 (2012)

2. PG Li Analysis of The Interface Damage of CRTSII Slab Track and Its Influnces. Dissertation, Southwest Jiaotong Univ, Chengdu,(2015)

3. FS Liu, ZP Zeng, B Wu Research on the CRTSII Slab Ballastless Track Buckling Deformation in the Process of Construction. J of Railw Eng Soc 1 (2015)

4. Sinohydro Bureau 7 CO.,LTD The Show on the Core Technology of the High-speed Railway about the Construction Technology of Ballastless Track System on Beijing-Shanghai High Speed Railway. Tinajin Science \& Technology Translation \& Publishing CO.,LTD, Tinajin (2011)

5. M Zhou, GL Dai, B Yan Analysis of gap and deformation of CRTSIItrack on bridges under temperature . J Railw Sci and Eng 13,12 (2016)

6. HW He Ballastless Track Technology. China Railway Publishing House, Beijing (2005)

7. Sinohydro Bureau 7 CO.,LTD Manufacture Technology of the CRTSII Ballastless Track Slab on Beijing-Shanghai High Speed Railway. Tinajin 
Science \& Technology Translation \& Publishing CO.,LTD, Tinajin (2009)

8. JTG D40-2011, Specifications for Design of Highway Cement Concrete Pavement

9. GB 50010-2010, Code for design of concrete structures

10. Y Liu. Study on Characteristics and Influences of CRTSII Slab Track Early Temperature Field . Dissertation, Southwest Jiaotong Univ, Chengdu (2013)

11. Q Yuan, JG Guo, DH Deng et al Experimental study on the bonding strength between high modulus cement emulsified asphalt for slab track and concrete. J Railw Sci and Eng 10,6 (2013)

12. $\mathrm{P}$ Wang, JL Xiao, R Chen et al Technology of Continuous Welded Rail on Bridges for High-speed Railway. China Railway Publishing House, Beijing (2016) 